\title{
Kinetics of the reactions of isoprene-derived hydroxynitrates: gas phase epoxide formation and solution phase hydrolysis
}

M. I. Jacobs et al.

Correspondence to: M. J. Elrod (mjelrod@oberlin.edu) 


\section{Synthesis of hydroxynitrates}

\subsection{Synthesis of 4-hydroxy-3-nitrooxy isoprene (4,3-HNI)}

The synthesis of 4,3-HNI required the preparation of 3,4-epoxyisoprene (2-(prop-1-en-2yl)oxirane), which was synthesized according to a procedure first reported by Harwood et al. (Harwood et al., 1990). In a 1-L round bottom flask, $60 \mathrm{~g}$ of powdered $\mathrm{NaOH}$ and $50 \mathrm{~g}$ of trimethylsulfonium methyl sulfate (Oakwood Products, Inc.) were added to $600 \mathrm{~mL}$ of $\mathrm{CH}_{2} \mathrm{Cl}_{2}$. The solution was vigorously stirred while $20 \mathrm{~mL}$ of methacrolein (Sigma-Aldrich) were added in $300 \mu \mathrm{L}$ increments every 3 minutes. The reaction mixture was washed with $200 \mathrm{~mL}$ of $\mathrm{DI} \mathrm{H}_{2} \mathrm{O}$ thrice to remove the solid $\mathrm{NaOH}$. Anhydous $\mathrm{MgSO}_{4}$ (roughly $5 \mathrm{~g}$ ) was added to the resulting organic phase to dry the solution. After filtering off the $\mathrm{MgSO}_{4}$, a majority of the solvent was removed at atmospheric pressure $\left(50^{\circ} \mathrm{C}\right.$ water bath) using a fractionating (Vigreux) column yielding approximately $20 \mathrm{~mL}$ of residue. Atmospheric pressure distillation was performed on the residue and the liquid ( $6.80 \mathrm{~g})$ with a boiling range of $65-80{ }^{\circ} \mathrm{C}$ was collected. $\mathrm{A}{ }^{1} \mathrm{H}$ NMR spectrum confirmed the presence and purity of the desired epoxide.

4,3-HNI was synthesized from 3,4-epoxy isoprene via reaction with fuming $\mathrm{HNO}_{3}$ (Muthuramu et al., 1993). In a 1-L round bottom flask, $2.75 \mathrm{~g}$ of 3,4-epoxyisoprene were added to $200 \mathrm{~mL}$ of $\mathrm{CH}_{2} \mathrm{Cl}_{2}$ and cooled to $0{ }^{\circ} \mathrm{C}$. Fuming $\mathrm{HNO}_{3}(10 \mathrm{~mL})$ was added drop-wise using a burette over the course of 20 minutes. After addition of $\mathrm{HNO}_{3}$, the reaction mixture was refluxed in a $50{ }^{\circ} \mathrm{C}$ water bath for 15 minutes. After refluxing, $\mathrm{NaHCO}_{3}$ was added slowly until all of the acid had been neutralized. The solid was filtered off and the vast majority of the solvent was removed under reduced pressure $\left(150-400 \mathrm{mbar}, 30^{\circ} \mathrm{C}\right)$. The residue was purified with flash chromatography using a 14-in. silica gel (Baker, $40 \mu \mathrm{m}$ packing, $60 \AA$ pore diameter) column, with a hexanes and ethyl acetate (3:2 ratio) eluent. Nitrate species were detected using 
diphenylamine as previously described (Treves et al., 2000). ${ }^{1} \mathrm{H}$ and ${ }^{13} \mathrm{C}$ NMR confirmed the presence and purity of 4,3-HNI. The final remaining solvent was removed using the vacuum system at low pressure ( 150 mtorr, room temperature), and the final product was stored at $-80{ }^{\circ} \mathrm{C}$.

\subsection{Synthesis of 1-hydroxy-4-nitrooxy isoprene (1,4-HNI)}

1,4-HNI was synthesized from 2-methyl-2-vinyloxirane (Sigma-Aldrich) via reaction with fuming $\mathrm{HNO}_{3}$ using a modified method to minimize the presence of water (Lockwood et al., 2010). A 1.5:1 molar ratio of 2-methyl-2-vinyloxirane to fuming $\mathrm{HNO}_{3}$ was used. In a 500$\mathrm{mL}$ round bottom flask, 2-methyl-2-vinyloxirane $(3.00 \mathrm{~g}, 35.7 \mathrm{mmol})$ was added to $200 \mathrm{~mL}$ of $\mathrm{CHCl}_{3}$ under a $\mathrm{N}_{2}$ flow at $0{ }^{\circ} \mathrm{C}$. Fuming $\mathrm{HNO}_{3}(1.00 \mathrm{~mL}, 24.0 \mathrm{mmol})$ was added drop-wise to the reaction flask over the course of 10 minutes. The remaining procedure matched that of 4,3HNI as described above, except a 2:1 hexane to ethyl acetate mobile phase was used for flash chromatography.

\subsection{Synthesis of 1-hydroxy-2-nitrooxybut-3-ene (1,2-HNB)}

1,2-HNB was synthesized using the 4,3-HNI and 1,4-HNI synthetic procedures detailed above. $2.0 \mathrm{~g}$ of 3,4-epoxy-1-butene (98\%, Sigma-Aldrich) was dissolved in $200 \mathrm{~mL} \mathrm{CH}_{2} \mathrm{Cl}_{2}$. $5.0 \mathrm{~mL}$ of fuming $\mathrm{HNO}_{3}$ was added drop-wise over the course of 15 minutes. The remaining procedures were identical to 1,4-HNI synthesis.

\subsection{NMR assignments}

All ${ }^{1} \mathrm{H}$ chemical shifts for deuterated chloroform and aqueous solutions were determined relative to the $\mathrm{CHCl}_{3}$ and $\mathrm{HDO}$ peaks at 7.26 and $4.79 \mathrm{ppm}$, respectively. The primary contaminant for the synthesized hydroxynitrates in each of the spectra is ethyl acetate, which was not removed entirely until gas phase experiments were performed. Peaks from ethyl acetate are 
labeled as EA while peaks from the relevant hydroxynitrate are labeled according to a numbering scheme where the isoprene backbone is numbered 1 through 4 (using the isoprene IUPAC naming scheme) with the methyl group being carbon number 5. The protons on each isomer are labeled a-e with proton "a" attached to carbon "1", proton "c" attached to carbon "3", etc. Each isomer is labeled on the ${ }^{1} \mathrm{H}$ and ${ }^{13} \mathrm{C}$ spectra for reference.

\subsubsection{4,3-HNI structure assignment}

${ }^{1} \mathrm{H}$ and ${ }^{13} \mathrm{C}$ spectra of 4,3-HNI were collected in $\mathrm{CDCl}_{3}$ and the annotated spectra are given below (Figures S1 and S2). The structural position of the nitrate group was confirmed using HMQC. The HMQC spectrum (Figure S3) shows that ${ }^{1} \mathrm{H}$ peak at $5.32 \mathrm{ppm}$ is a nitrate proton and not an alkene proton due to the coupling with the ${ }^{13} \mathrm{C}$ peak at $86.69 \mathrm{ppm}$ (which is too low for an alkene carbon). The triplet and doublet splitting patterns of the nitrate peak and the alcohol peak (at $3.78 \mathrm{ppm}$ ), respectively, confirms that the secondary nitrate was exclusively formed in the synthetic procedure. The final ${ }^{1} \mathrm{H}$ and ${ }^{13} \mathrm{C}$ assignments for $4,3-\mathrm{HNI}$ are presented in Table S1. 


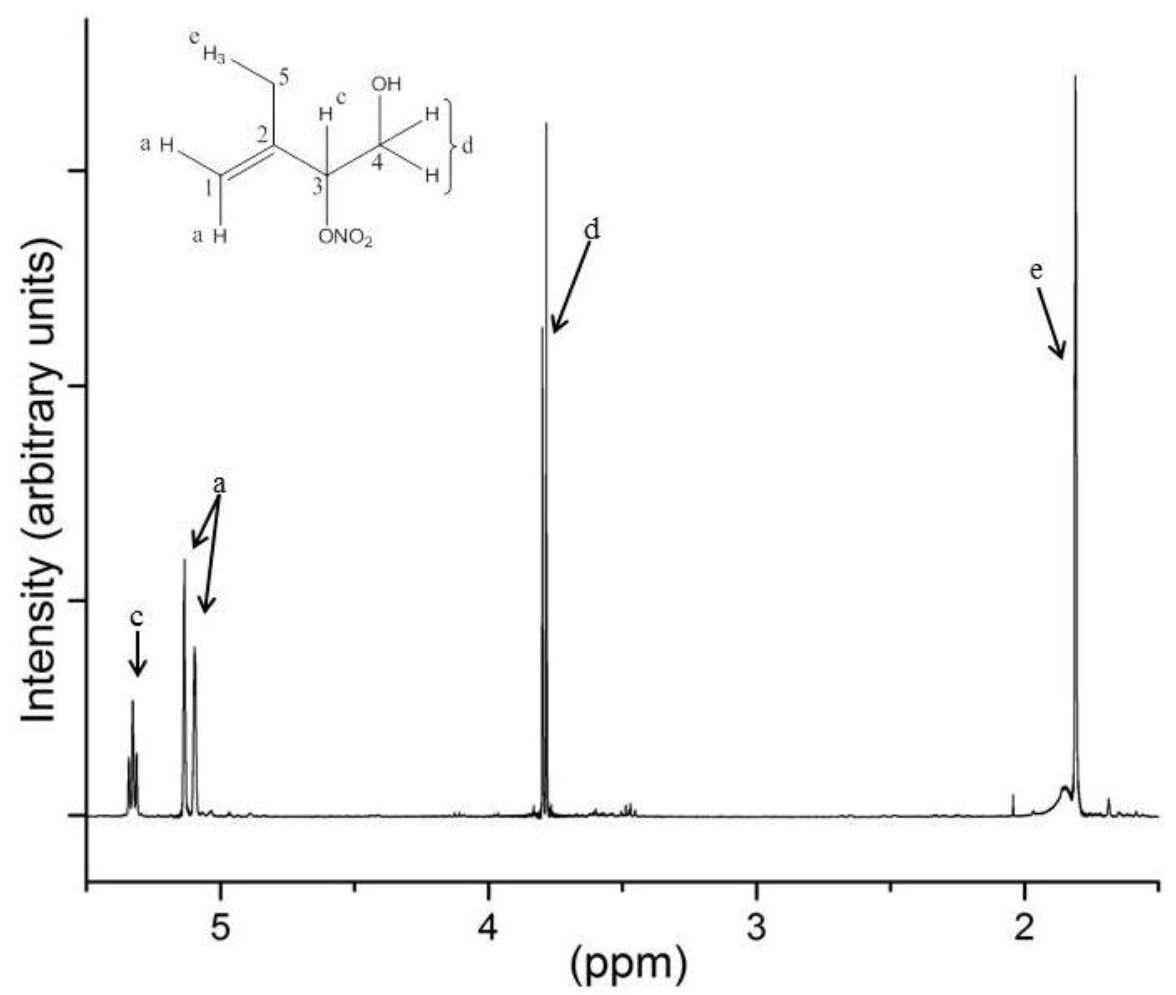

Figure S1. ${ }^{1} \mathrm{H}$ NMR spectrum of $4,3-\mathrm{HNI}$ collected in $\mathrm{CDCl}_{3}$.

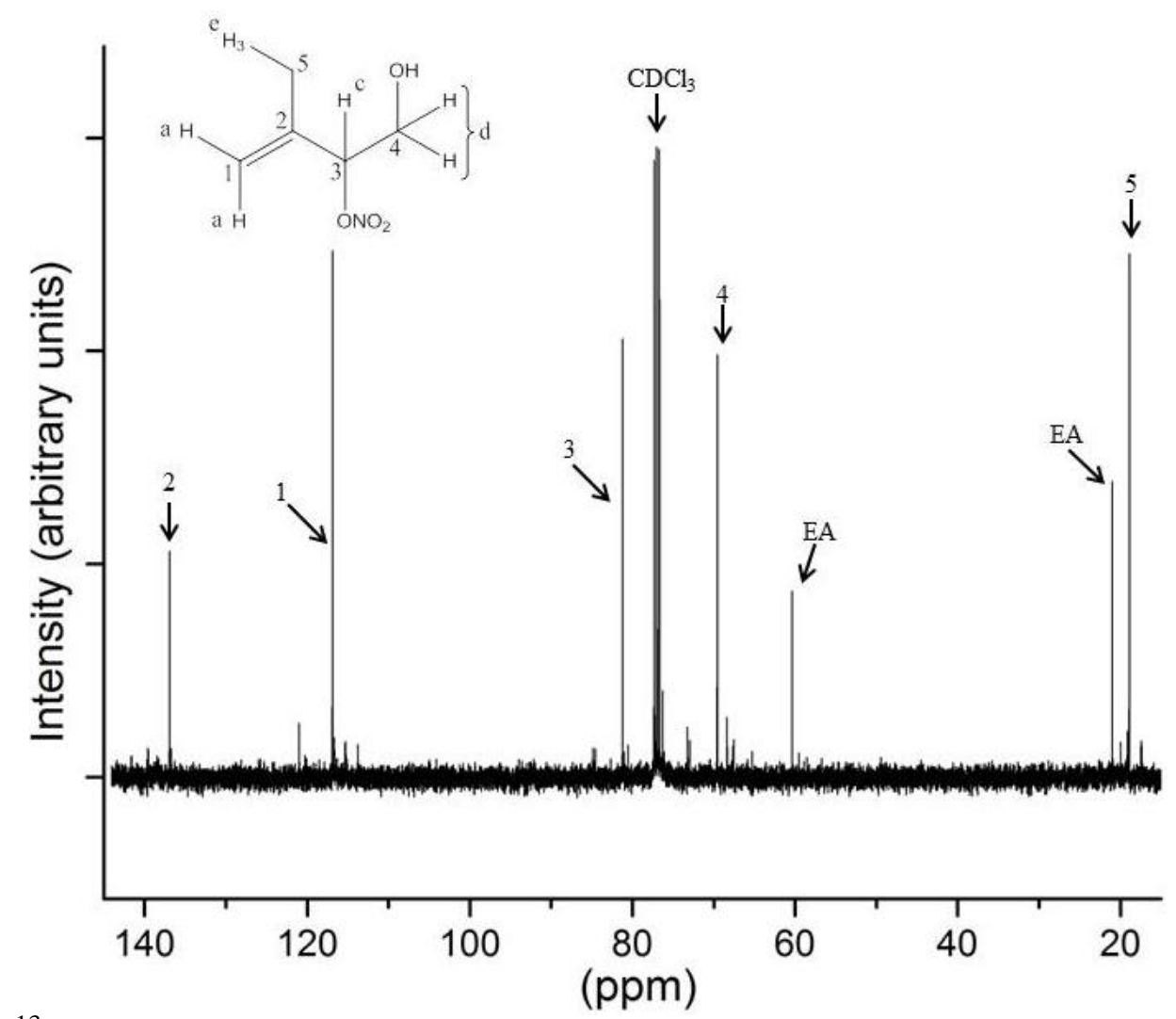

Figure S2. ${ }^{13} \mathrm{C}$ NMR spectrum of 4,3-HNI collected in $\mathrm{CDCl}_{3}$ (EA indicates ethyl acetate solvent impurity). 


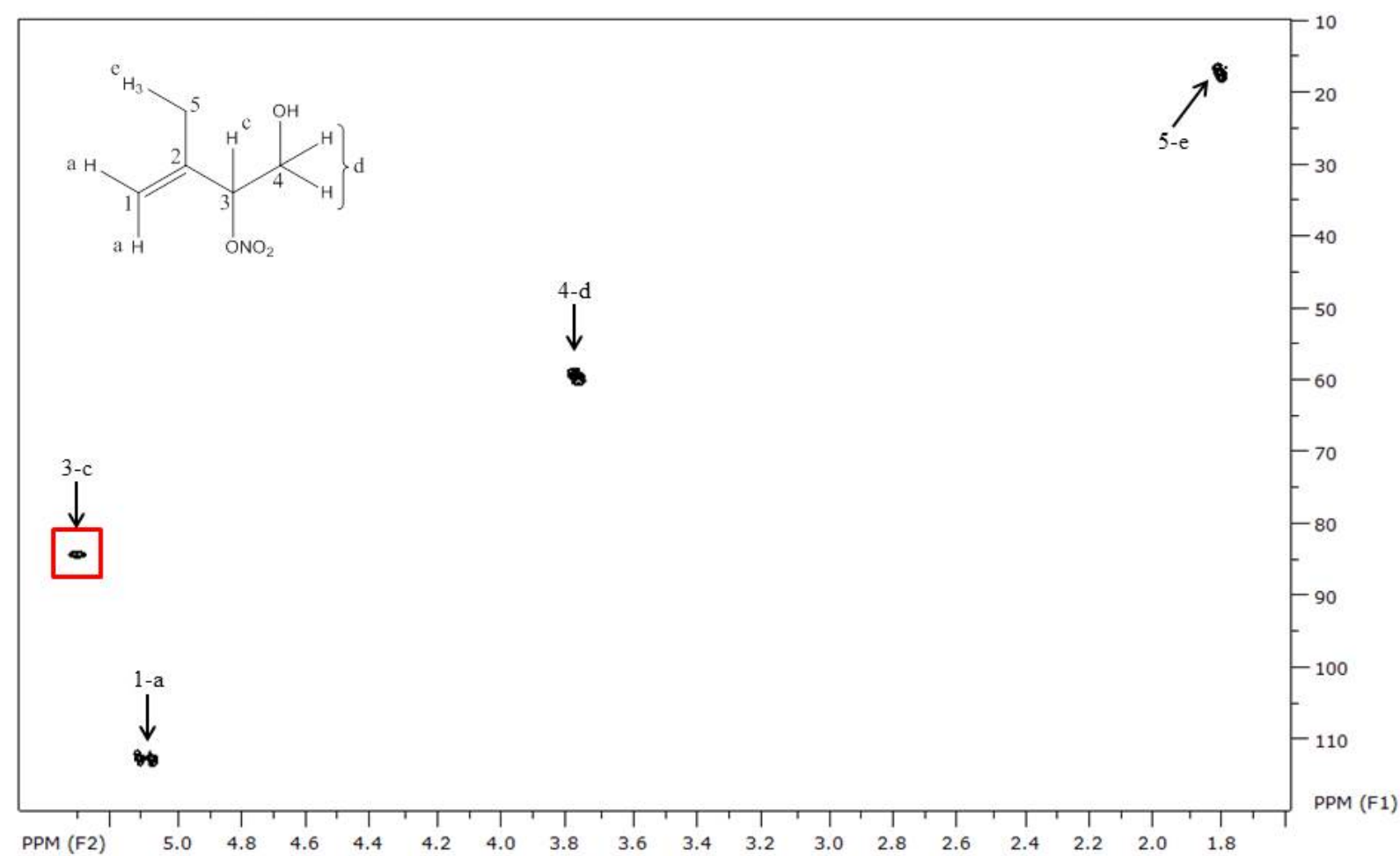

Figure S3. HMQC NMR spectrum of 4,3-HNI collected in $\mathrm{CDCl}_{3}$. The red box indicates the coupling between peaks at $5.32 \mathrm{ppm}$ in the ${ }^{1} \mathrm{H}$ spectrum and $86.69 \mathrm{ppm}$ from the ${ }^{13} \mathrm{C}$ spectrum, which confirms those carbon and hydrogen nuclei as being adjacent to the nitrate functional group.

\subsubsection{E and Z 1,4-HNI structure assignment}

${ }^{1} \mathrm{H}$ and ${ }^{13} \mathrm{C}$ spectra of $1,4-\mathrm{HNI}$ were collected in $\mathrm{CDCl}_{3}$ and the annotated spectra are given below (Figures S4 and S5). The structural position of the nitrate group was once again confirmed using HMQC. The HMQC spectrum (Figure S6) shows that the ${ }^{1} \mathrm{H}$ peak at $5.00 \mathrm{ppm}$ is a nitrate proton and not an alkene proton due to the coupling with the ${ }^{13} \mathrm{C}$ peaks at 69.3 and $68.9 \mathrm{ppm}$ (which are too low for an alkene carbon). Furthermore, a COSY spectrum (Figure S7) showed coupling between the ${ }^{1} \mathrm{H}$ nitrate peak at $5.00 \mathrm{ppm}$ and the ${ }^{1} \mathrm{H}$ alkene peaks at 5.46 and $5.65 \mathrm{ppm}$, which demonstrates the nitrate group is adjacent to an alkene carbon atom. The fact that no other peaks are observed indicates that the other possible isomers (e.g. 2,1- and 1,2-HNI) were not formed. Because the two isomers were not synthesized in equal yields, the assignment of the $\mathrm{E}$ vs. $\mathrm{Z}$ isomer was made using the relative sizes of the peaks - primarily E-1,4-HNI was 
formed, so the larger product peaks were attributed to E-1,4-HNI. The final assignments for (E) 1,4-HNI and (Z) 1,4-HNI are presented in Table S1.

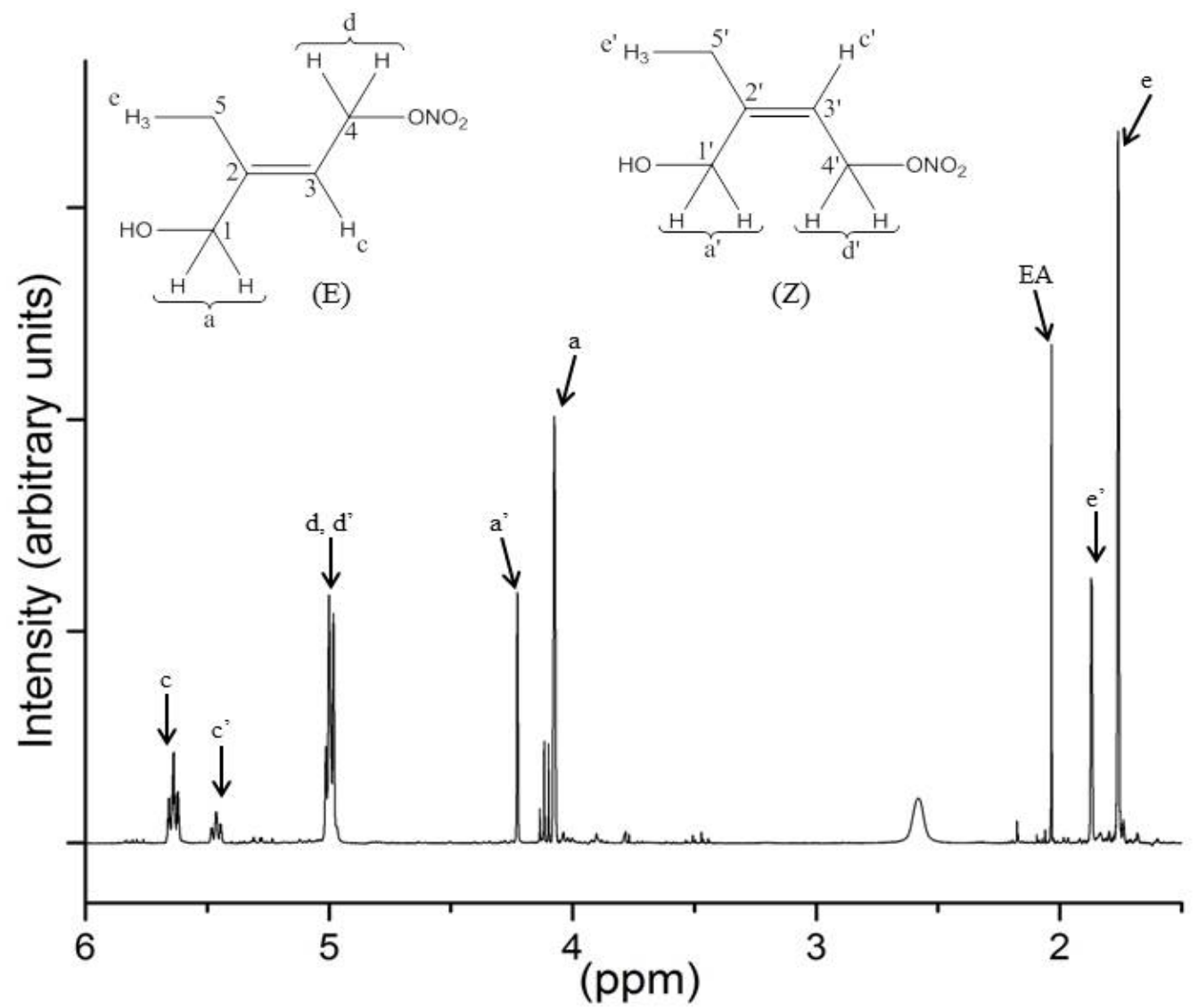

Figure S4. ${ }^{1} \mathrm{H}$ NMR spectrum of (E,Z) 1,4-HNI collected in $\mathrm{CDCl}_{3}$ 


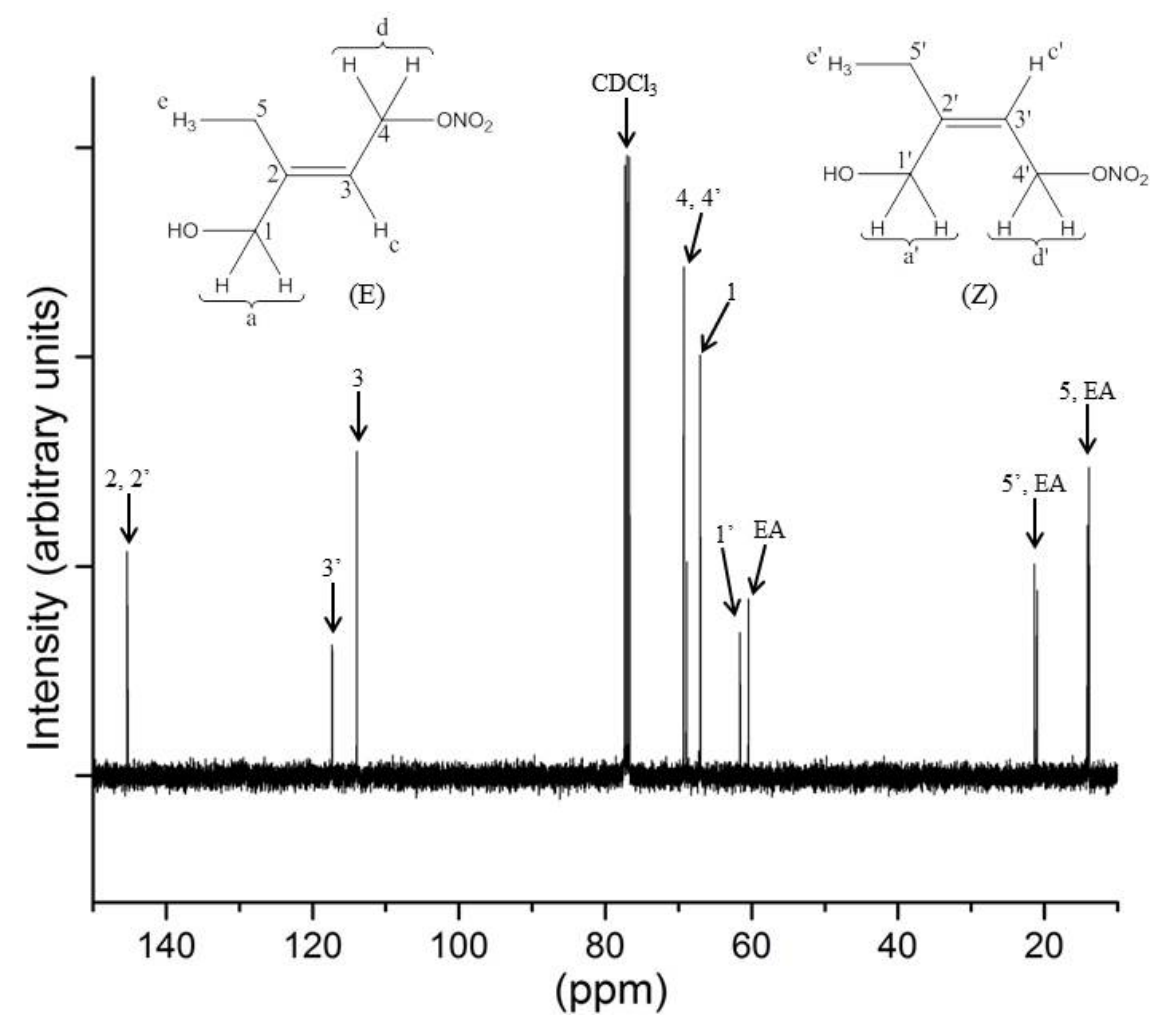

Figure S5. ${ }^{13} \mathrm{C}$ NMR spectrum of (E,Z) 1,4-HNI collected in $\mathrm{CDCl}_{3}$

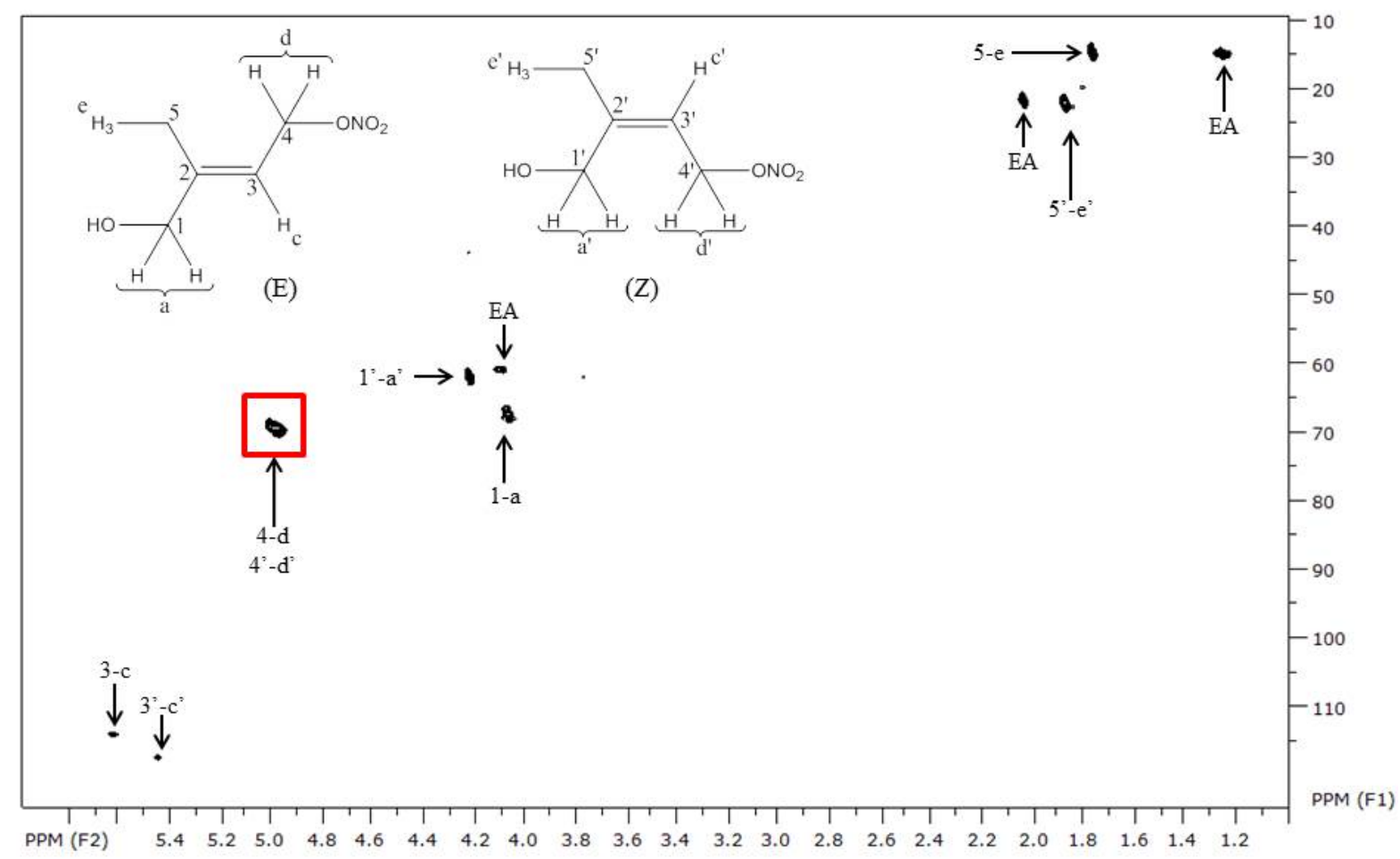

Figure S6. HMQC NMR spectrum of $\mathrm{E}$ and $\mathrm{Z} 1,4-\mathrm{HNI}$ collected in $\mathrm{CDCl}_{3}$. The red box indicates the coupling between peaks at $5.00 \mathrm{ppm}$ in the ${ }^{1} \mathrm{H}$ spectrum and 68.9 and $69.3 \mathrm{ppm}$ from the ${ }^{13} \mathrm{C}$ spectrum, which confirms those carbon and hydrogen nuclei as being adjacent to the nitrate functional group. 


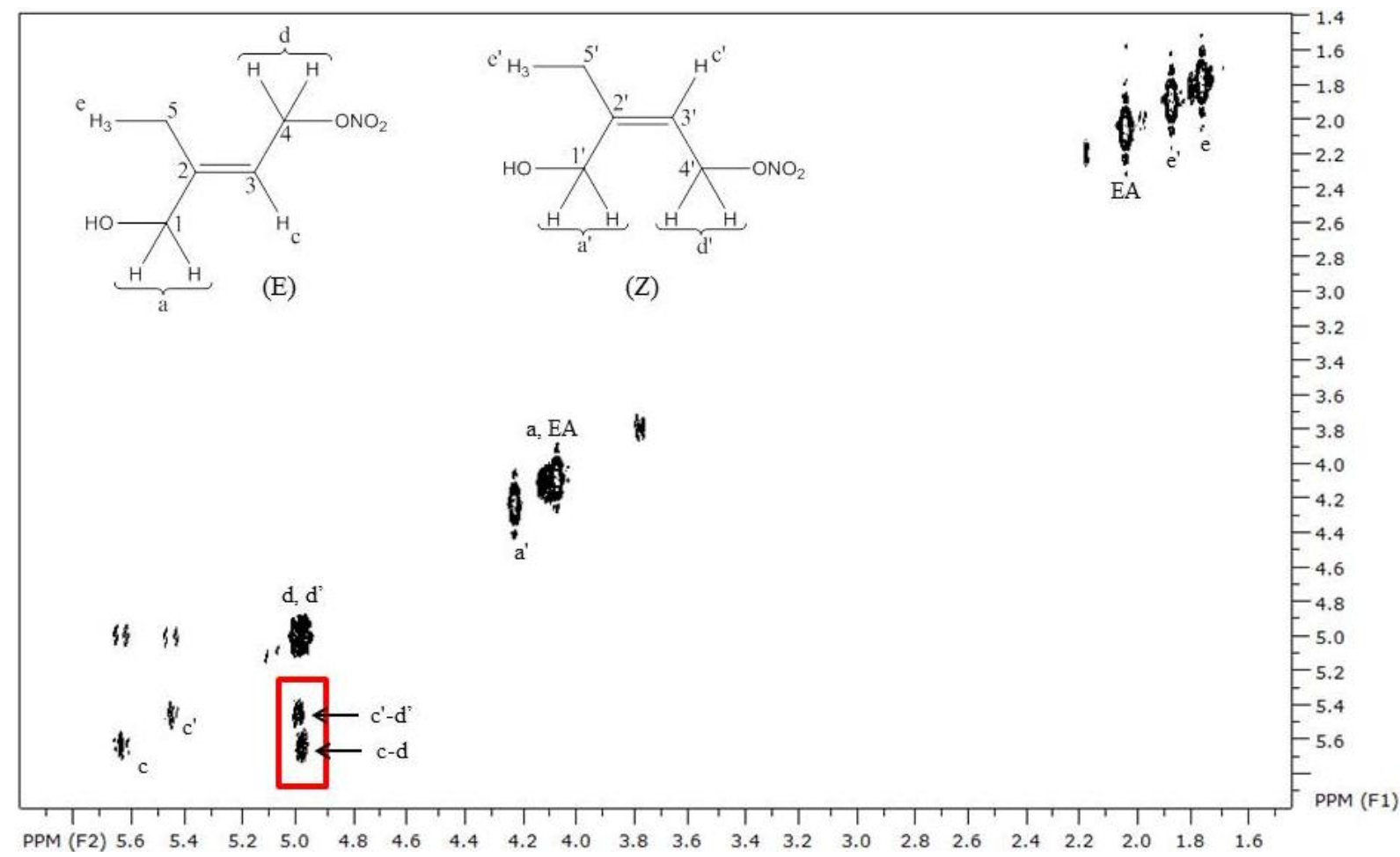

Figure S6. COSY NMR spectrum of $\mathrm{E}$ and Z 1,4-HNI collected in $\mathrm{CDCl}_{3}$. The red box indicates the coupling between the $5.00 \mathrm{ppm}{ }^{1} \mathrm{H}$ peaks and the 5.46 and $5.65 \mathrm{ppm}{ }^{1} \mathrm{H}$ peaks, which demonstrates the nitrate group is adjacent to an alkene carbon atom in both of the isomers.

\begin{tabular}{cccccccc}
\hline \multirow{2}{*}{ H label } & \multirow{2}{*}{ C label } & \multicolumn{2}{c}{$4,3-\mathrm{HNI}$} & \multicolumn{2}{c}{$(\mathrm{E}) \mathrm{1,4- \textrm {HNI }}$} & \multicolumn{2}{c}{$(\mathrm{Z}) 1,4-\mathrm{HNI}$} \\
& & ${ }^{1} \mathrm{H}$ & ${ }^{13} \mathrm{C}$ & ${ }^{1} \mathrm{H}$ & ${ }^{13} \mathrm{C}$ & ${ }^{1} \mathrm{H}$ & ${ }^{13} \mathrm{C}$ \\
\hline $\mathrm{A}$ & 1 & $5.10(\mathrm{ds})$ & 115.16 & $4.07(\mathrm{~s})$ & 67.11 & $4.22(\mathrm{~s})$ & 61.64 \\
& 2 & & 138.52 & & 145.21 & & 145.30 \\
$\mathrm{C}$ & 3 & $5.32(\mathrm{t})$ & 86.69 & $5.65(\mathrm{t})$ & 114.03 & $5.46(\mathrm{t})$ & 117.36 \\
$\mathrm{D}$ & 4 & $3.78(\mathrm{~d})$ & 61.69 & $5.00(\mathrm{~d})$ & 68.88 & $5.00(\mathrm{~d})$ & 69.28 \\
$\mathrm{E}$ & 5 & $1.88(\mathrm{~s})$ & 19.18 & $1.76(\mathrm{~s})$ & 13.94 & $1.87(\mathrm{~s})$ & 21.38 \\
\hline
\end{tabular}

Table S1. ${ }^{1} \mathrm{H}$ and ${ }^{13} \mathrm{C} \mathrm{NMR}$ (in $\mathrm{CDCl}_{3}$ solvent) assignments for all three of the synthesized isoprene derived hydroxynitrates.

A previous study has reported the synthesis of 1,2-HNI (a tertiary nitrate) and 2,1-HNI using a similar procedure as that reported here (Lockwood et al., 2010). The mechanism for the four possible hydroxynitrate isomers from 2-methyl-2-vinyloxirane is provided in Figure S7.

Previous work from our laboratory has demonstrated that hydrolysis of the tertiary nitrate occurs rapidly (with a lifetime of only a couple of minutes) and because of this, isolating the tertiary 
nitrate using conventional chromatographic techniques is expected to be very difficult (Darer et al., 2011; Hu et al., 2011). The hydroxynitrate synthetic procedures reported herein were designed to minimize the amount of water added to the reaction to avoid hydrolysis of the products. Nonetheless, based on the NMR data collected, we did not detect the 1,2- nor 2,1-HNI isomers at any point in the synthetic process.

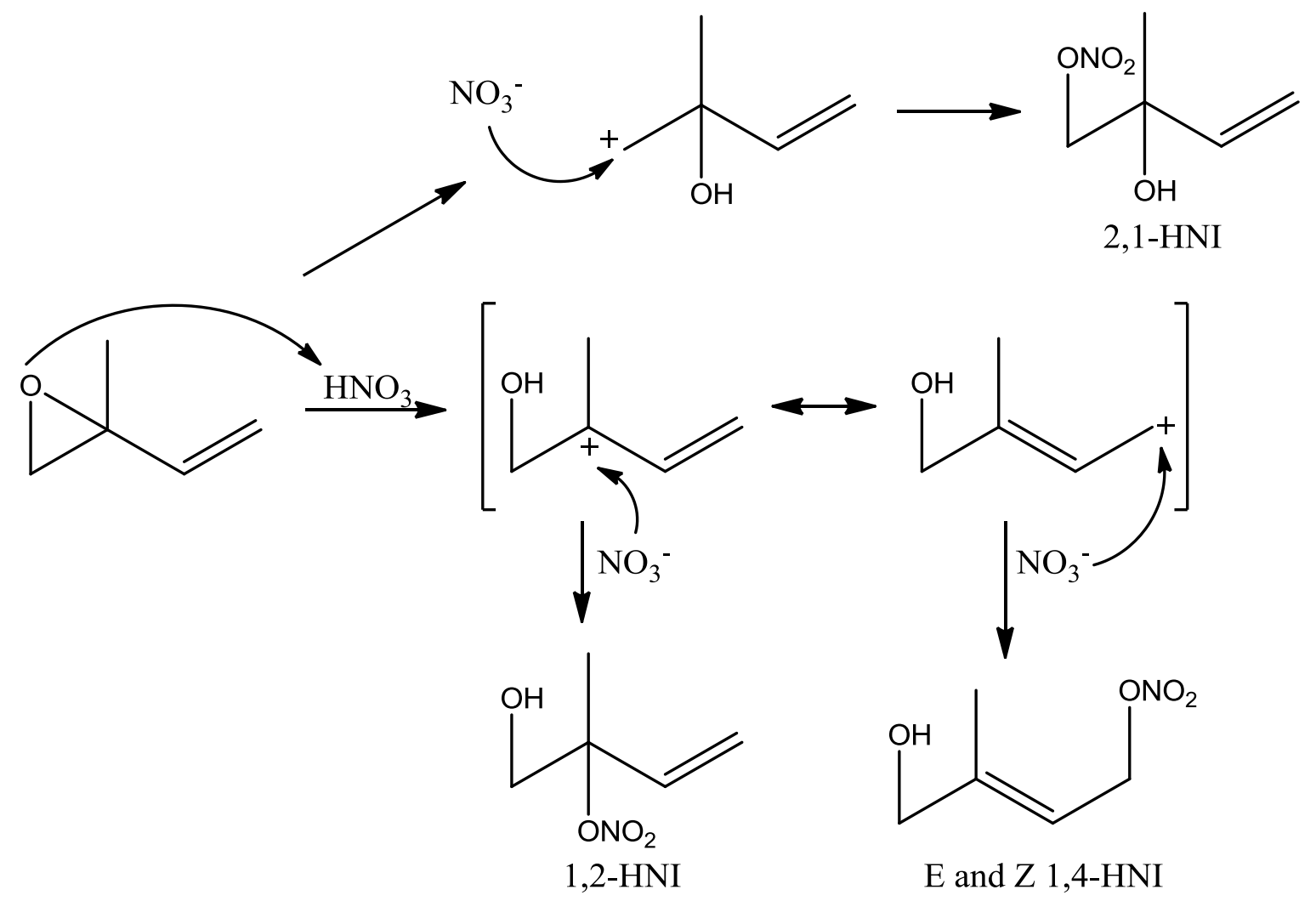

Figure S7. Mechanism for E and Z 1,4-HNI and 1,2-HNI synthesis from reaction of 2-methyl-2vinyloxirane with fuming nitric acid.

\subsubsection{1,2-HNB assignment}

${ }^{1} \mathrm{H}$ and ${ }^{13} \mathrm{C}$ spectra of $1,2-\mathrm{HNB}$ were collected in $\mathrm{CDCl}_{3}$ and the annotated spectra are given below (Figures S8 and S9). The peak assignments for 1,2-HNB were made based on the NMR spectra for the other two hydroxynitrates, and the specific shifts are given in Table S2. 


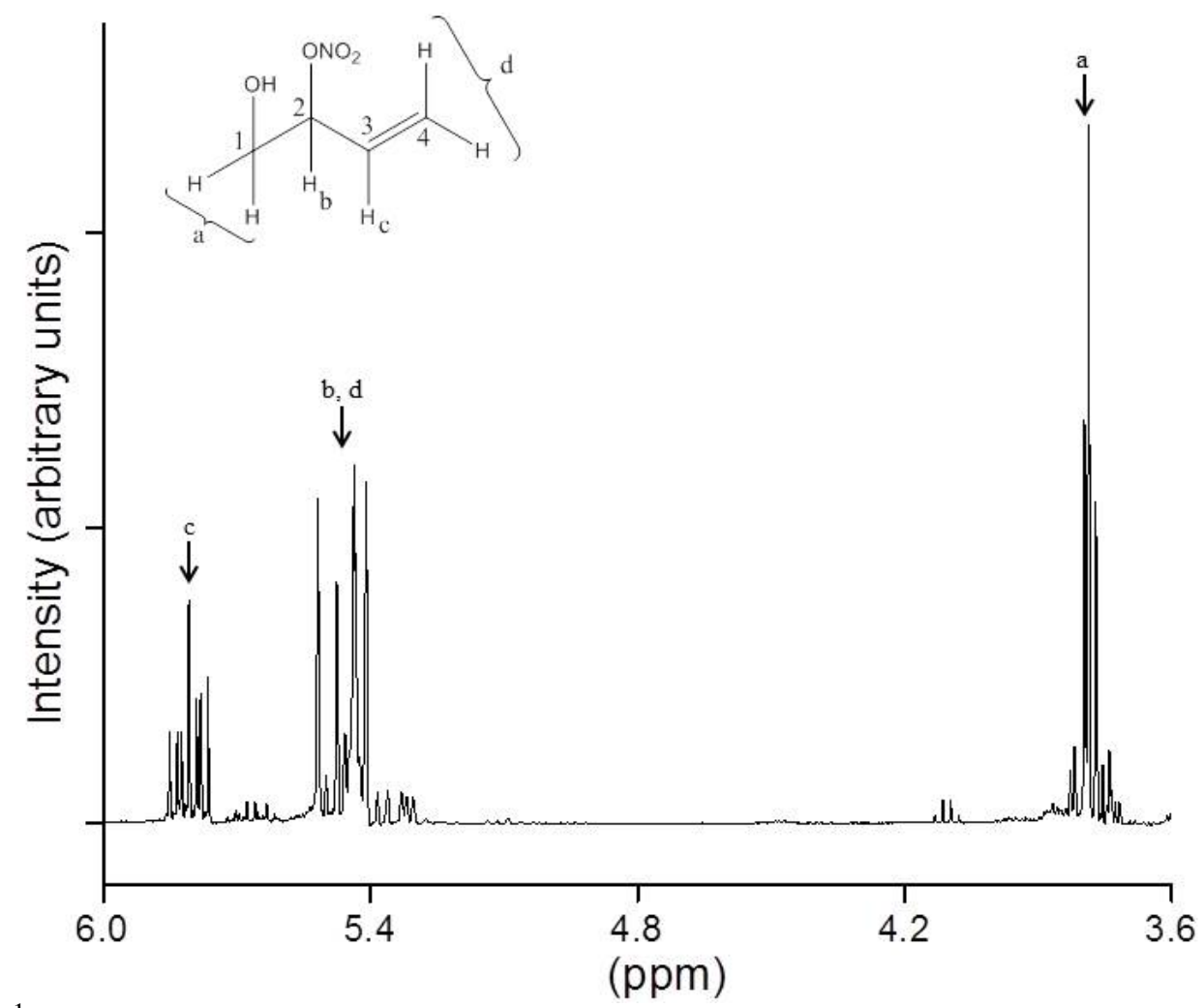

Figure S8. ${ }^{1} \mathrm{H}$ NMR spectrum of $1,2-\mathrm{HNB}$ collected in $\mathrm{CDCl}_{3}$

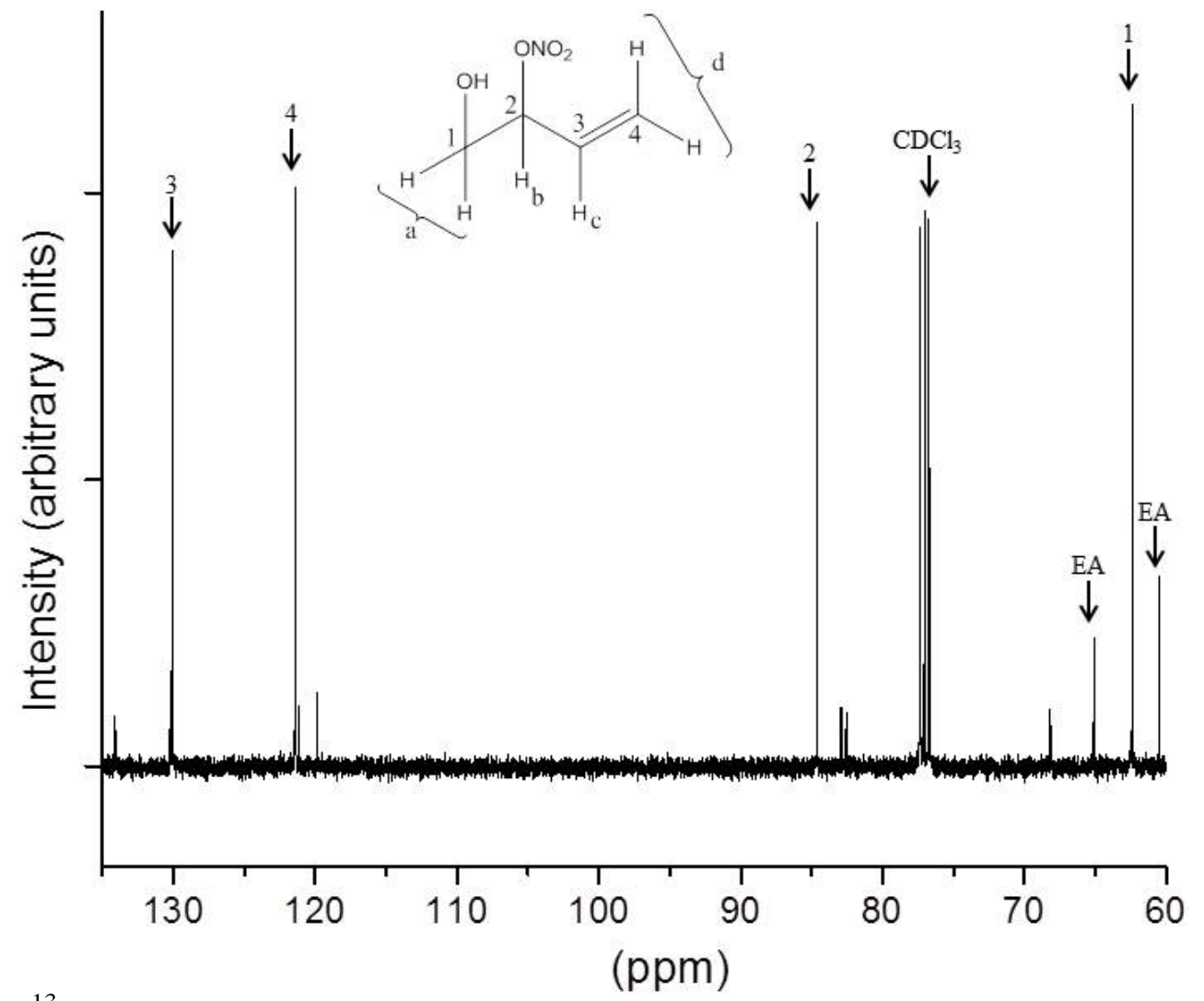

Figure S9. ${ }^{13} \mathrm{C}$ NMR spectrum of $1,2-\mathrm{HNB}$ collected in $\mathrm{CDCl}_{3}$ 


\begin{tabular}{cccc}
\hline \multirow{2}{*}{ H label } & \multirow{2}{*}{ C label } & ${ }^{1} \mathrm{H}$ & ${ }^{13} \mathrm{C}$ \\
\hline $\mathrm{a}$ & 1 & 3.81 & 62.44 \\
$\mathrm{~b}$ & 2 & 5.45 & 84.57 \\
$\mathrm{c}$ & 3 & 5.85 & 130.11 \\
$\mathrm{~d}$ & 4 & 5.47 & 121.56 \\
\hline
\end{tabular}

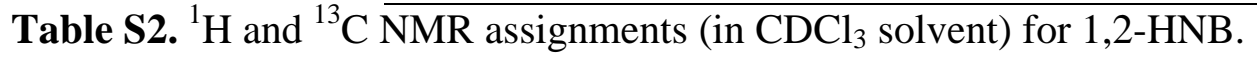

\section{References}

Darer, A. I., Cole-Filipiak, N. C., O’Connor, A. E. and Elrod, M. J.: Formation and stability of atmospherically relevant isoprene-derived organosulfates and organonitrates., Environ. Sci. Technol., 45, 1895-902, doi:10.1021/es103797z, 2011.

Harwood, L. M., Casy, G. and Sherlock, J.: A Simple Laboratory Procedure for Preparation of (1-methylethenyl)oxirane (3,4-epoxyisoprene), Synth. Commun., 20(9), 1287-1292, 1990.

Hu, K. S., Darer, A. I. and Elrod, M. J.: Thermodynamics and kinetics of the hydrolysis of atmospherically relevant organonitrates and organosulfates, Atmos. Chem. Phys., 11, 83078320, doi:10.5194/acp-11-8307-2011, 2011.

Lockwood, A. L., Shepson, P. B., Fiddler, M. N. and Alaghmand, M.: Isoprene nitrates: preparation, separation, identification, yields, and atmospheric chemistry, Atmos. Chem. Phys., 10, 6169-6178, doi:10.5194/acp-10-6169-2010, 2010.

Muthuramu, K., Shepson, P. B., Street, K. and York, N.: Preparation, Analysis, and Atmospheric Production of Multifunctional Organic Nitrates, Environ. Sci. Technol., 27, 1117-1124, 1993.

Treves, K., Shragina, L. and Rudich, Y.: Henry's Law Constants of Some $\beta$-, $\gamma$-, and $\delta$ - Hydroxy Alkyl Nitrates of Atmospheric Interest, Environ. Sci. Technol., 34, 1197-1203, 2000. 